\section{BRIEF REPORT}

\title{
Oseltamivir Resistance during Treatment of Influenza A (H5N1) Infection
}

\author{
Menno D. de Jong, M.D., Ph.D., Tran Tan Thanh, M.Sc., \\ Truong Huu Khanh, M.D., Vo Minh Hien, M.D., Gavin J.D. Smith, Ph.D., \\ Nguyen Vinh Chau, M.D., Bach Van Cam, M.D., Phan Tu Qui, M.D., \\ Do Quang Ha, M.D., Ph.D., Yi Guan, M.D., Ph.D., J.S. Malik Peiris, D.Phil., M.D., \\ Tran Tinh Hien, M.D., Ph.D., and Jeremy Farrar, D.Phil., F.R.C.P.
}

SUMMARY

Influenza A (H5N1) virus with an amino acid substitution in neuraminidase conferring high-level resistance to oseltamivir was isolated from two of eight Vietnamese patients during oseltamivir treatment. Both patients died of influenza A (H5N1) virus infection, despite early initiation of treatment in one patient. Surviving patients had rapid declines in the viral load to undetectable levels during treatment. These observations suggest that resistance can emerge during the currently recommended regimen of oseltamivir therapy and may be associated with clinical deterioration and that the strategy for the treatment of influenza A (H5N1) virus infection should include additional antiviral agents.

I

NFLUENZA A (H5N1) VIRUS CAUSES SEVERE DISEASE IN HUMANS AND POSES an unprecedented pandemic threat. ${ }^{1-3}$ The neuraminidase inhibitor oseltamivir constitutes an important treatment option, and stockpiling of this drug is part of pandemic-preparedness plans. ${ }^{4}$ However, data on the efficacy and development of drug resistance in human influenza A (H5N1) virus are scarce. We report the isolation of oseltamivir-resistant influenza A (H5N1) variants from two patients who died of the infection, in one case despite the early initiation of treatment. Furthermore, we provide evidence suggesting that the presence of detectable virus after the completion of treatment is associated with a poor outcome. These observations have implications for the treatment of influenza A (H5N1) virus infection.

\section{CASE REPORT}

A previously healthy 13-year-old Vietnamese girl weighing $28 \mathrm{~kg}$ (Patient 1 in Table 1) presented to a hospital in Dong Thap Province on January 22, 2005, with a one-day history of fever and cough. The day before, her mother (Patient 2 in Table 1) had died of influenza A (H5N1) virus infection after one day of oseltamivir treatment. Virus isolated from the mother did not reveal oseltamivir-resistance mutations. Because influenza A (H5N1) virus infection was suspected in the child at presentation, she received an initial 75-mg dose of oseltamivir and was transferred to a pediatric referral hospital. On admission, she had a temperature of $40.3^{\circ} \mathrm{C}$, a pulse of 106 beats per minute, a respiratory rate of 36 breaths per minute, and normal blood pressure. Results of physical examination and routine biochemical measurements were unre-
From the Oxford University Clinical Research Unit, Hospital for Tropical Diseases (M.D.J., T.T.T., D.Q.H., J.F.), Pediatric Hospital Number One (T.H.K., B.V.C., P.T.Q.), and the Hospital for Tropical Diseases (V.M.H., N.V.C., T.T.H.) - all in Ho Chi Minh City, Vietnam; and the Department of Microbiology, University of Hong Kong, Queen Mary Hospital, Hong Kong Special Administrative Region, China (G.J.D.S., Y.G., J.S.M.P.). Address reprint requests to Dr. de Jong at the Oxford University Clinical Research Unit, Hospital for Tropical Diseases, 190 Ben Ham Tu, District 5, Ho Chi Minh City, Vietnam, or at mddejong@hcm.vnn.vn.

N Engl J Med 2005;353:2667-72. Copyright $(2005$ Massachusetts Medical Society. 


\begin{tabular}{|c|c|c|c|c|c|c|}
\hline \multirow[t]{2}{*}{ Patient } & $\begin{array}{c}\text { Age }(y r) / \\
\text { Sex }\end{array}$ & Admission & & $\begin{array}{l}\text { Virus Detectable } \\
\text { at End of } \\
\text { Treatment }\end{array}$ & $\begin{array}{l}\mathrm{H} 274 \mathrm{Y} \text { in } \mathrm{Nl} \\
\text { at End of } \\
\text { Treatment' }\end{array}$ & Clinical Outcome \\
\hline & & Date & $\begin{array}{l}\text { Day of } \\
\text { Illness }\end{array}$ & & & \\
\hline 1 & $13 / F$ & January 2005 & 2 & Yes & Yes & Died on 8th day of illness \\
\hline $2 \xi$ & $35 / F$ & January 2005 & 6 & NA & NA & Died on 7th day of illness \\
\hline 3 & $16 / F$ & December 2004 & 7 & Yes & NA & Died on 20th day of illness \\
\hline 4 & $18 / F$ & January 2005 & 6 & Yes & Yes & Died on 20th day of illness \\
\hline 5 & $26 / F$ & January 2005 & 4 & NA & NA & Survived \\
\hline $6 \pi$ & $8 / F$ & January 2004 & 8 & No & - & Survived \\
\hline $7 \mathbb{8}$ & $23 / \mathrm{M}$ & February 2004 & 7 & No & - & Survived \\
\hline 8 & $22 / \mathrm{M}$ & February 2004 & 6 & No & - & Survived \\
\hline
\end{tabular}

* All patients started oseltamivir treatment on the day of admission.

$\uparrow$ NA denotes not applicable owing to insufficient follow-up.

\pm Patient 2 was the mother of Patient 1.

$\int$ This patient has been described previously. ${ }^{2}$

markable. Hematologic measurements showed a white-cell count of 4800 cells per cubic millimeter (normal range, 5500 to 15,500), with 12 percent lymphocytes, and a platelet count of 183,000 cells per cubic millimeter (normal range, 250,000 to $550,000)$. A blood culture showed no growth. A chest radiograph revealed a small focal pulmonary infiltrate in the right middle lobe (Fig. 1A).

The patient received a second 75-mg dose of oseltamivir within 6 hours after the first, followed by a third dose within the first 24 hours after admission. Treatment was then continued for four days at the standard dose of $75 \mathrm{mg}$ twice daily. Antibiotic treatment with ceftriaxone and amikacin was also given. During the first three days after admission, the patient remained in stable condition and did not require supplemental oxygen. A chest radiograph obtained on January 24 showed minimal progression of the infiltrate (Fig. 1B). At that time, her white-cell count was 3100 cells per cubic millimeter, with 26 percent lymphocytes.

On January 25 (the fourth day of oseltamivir treatment), the child's respiratory condition worsened and supplemental high-dose oxygen was given, initially by nasal cannula and later by continuous positive airway pressure. Antibiotic treatment was switched to vancomycin, ciprofloxacin, and amikacin. At this time, the pneumonia involved most of the right middle zone (Fig. 1C). A chest radiograph obtained on January 26 showed fur- ther progression of the infiltrate (Fig. 1D). Hematologic and biochemical measurements revealed a white-cell count of 1800 cells per cubic millimeter, with 41 percent lymphocytes; a platelet count of 97,000 cells per cubic millimeter; and increased serum alanine aminotransferase and aspartate aminotransferase levels (144 and 279 U per liter, respectively; normal range, less than 55 and less than 50 , respectively). Her respiratory condition continued to worsen, and she was intubated and ventilation was begun on January 27. A chest radiograph obtained on January 28 showed pneumonia involving the entire right lung and extension to the left lung (Fig. 1E). She died the same day. No autopsy was performed.

\section{METHODS}

\section{PATIENTS AND CLINICAL SPECIMENS}

We examined sequential pharyngeal swabs from Patient 1 and seven additional patients with influenza A (H5N1) infection from whom at least one pharyngeal swab obtained before treatment and during treatment with oseltamivir was available. The swabs were collected in viral-transport medium and stored at $-80^{\circ} \mathrm{C}$.

\section{VIROLOGIC INVESTIGATIONS}

Virus isolation was performed in Madin-Darby canine-kidney cells in biosafety level III culture facilities, and influenza viruses were identified 


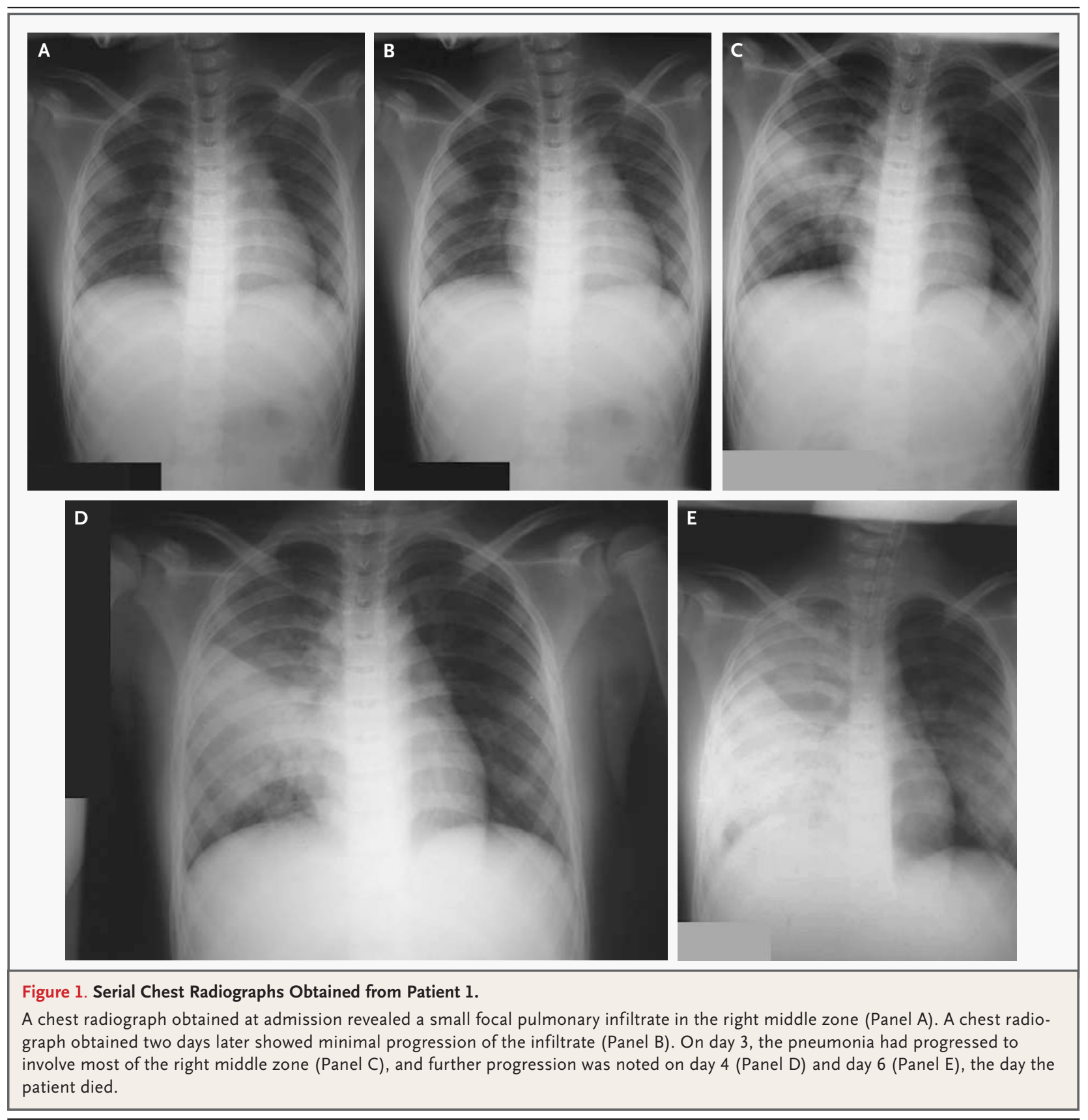

by serotype-specific reverse-transcriptase-polymerase-chain-reaction (RT-PCR) assays and hemagglutination-inhibition assays, as described previously. ${ }^{2,5,6}$ For the diagnosis of influenza A (H5N1) infection, nucleic acids were purified from $100 \mu \mathrm{l}$ of pharyngeal specimens and subtype-specific RT-PCR assays were performed as described previously. $2,7,8$ Quantitation of influenza A RNA was performed with the use of a real-time RT-PCR assay as described previously. ${ }^{6}$ The limit of detec-

tion of this assay is 1000 copies of RNA per milliliter. Quantitative analyses of serial pharyngeal specimens were performed in a batch-wise fashion. Positive and negative controls were included in all tests.

\section{SEQUENCE ANALYSIS OF NEURAMINIDASE GENES}

The sequence of the neuraminidase gene of initial viral isolates was analyzed as described previously, ${ }^{9}$ with the use of a BigDye Terminator 


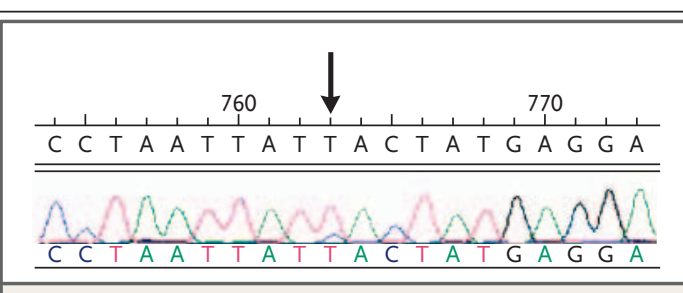

Figure 2. Sequencing Trace of the Neuraminidase Gene of Influenza A (H5N1) Virus Isolated from Patient 1 on the Fourth Day of Oseltamivir Treatment.

The arrow indicates the dominant $\mathrm{T}$ peak, with a smaller $\mathrm{C}$ peak at nucleotide position 763. A C $\rightarrow \mathrm{T}$ mutation at this position results in the substitution of tyrosine (encoded by TAC) for histidine (encoded by CAC) at amino acid position 274 , which confers resistance to oseltamivir in Nl-subtype influenza viruses.

Cycle Sequencing Kit (version 3.1, Applied Biosystems) on an ABI PRISM 3700 DNA Analyzer (Applied Biosystems). Sequence fragments were assembled and edited with the use of Lasergene software (version 6.0, DNASTAR). For direct sequencing of viral RNA from clinical specimens and follow-up isolates, a 200-bp fragment of N1 encompassing amino acid position 274 was amplified (primers available on request) and sequenced with the use of a CEQ Dye Terminator Cycle Sequencing Kit on a CEQ8000 system (Beckman Coulter). Alignment and residue analyses were performed with the use of BioEdit software (version 7). GenBank accession numbers of $\mathrm{N} 1$ sequences are shown in the Supplementary Appendix (available with the full text of this article at www.nejm.org).

RESULTS

ISOLATION OF OSELTAMIVIR-RESISTANT INFLUENZA A (H5N1) VARIANTS FROM PATIENT I Influenza A (H5N1) virus infection was diagnosed in another local laboratory by RT-PCR assay of a pharyngeal swab obtained at admission. This specimen was not available for further analysis. In our laboratory, influenza A (H5N1) virus was isolated from a throat swab obtained from Patient 1 on the fourth day of oseltamivir treatment (January 25,2005 ). Sequence analysis of the neuraminidase gene revealed the substitution of tyrosine for histidine at amino acid position 274 (H274Y), associated with high-level resistance to oseltamivir in influenza (N1) viruses. ${ }^{10}$ Analysis of the raw sequencing traces revealed the presence of a minor subpopulation of wild-type $274 \mathrm{H}$ variants among predominating $274 \mathrm{Y}$ mutants (Fig. 2). Virus was also isolated from a throat specimen obtained on January 28, 2005, two days after the completion of treatment. Sequence analyses of this strain as well as of viral RNA extracted directly from the swab also revealed the $\mathrm{H} 274 \mathrm{Y}$ change in N1. Although sequencing traces also revealed the presence of a minor wild-type $274 \mathrm{H}$ population in viral RNA from the swab, only $274 \mathrm{Y}$ variants were observed in the isolate, possibly reflecting overgrowth of the predominant mutant population during culture. Determination of influenza A (H5N1) RNA levels showed that the viral load had increased in the second specimen (Fig. 3).

\section{VIROLOGIC RESPONSES AND ISOLATION} OF A SECOND OSELTAMIVIR-RESISTANT STRAIN

Of 13 patients with RT-PCR-confirmed influenza A (H5N1) infection who were admitted to the Hospital for Tropical Diseases in Ho Chi Minh City, Vietnam, between January 2004 and February 2005, at least one throat swab obtained before treatment and during treatment was available from 7 patients (Patients 2 through 8 in Table 1; additional information on clinical features at admission, treatment, and outcomes is available in the Supplementary Appendix). Treatment with oseltamivir at the recommended dose and duration $(75 \mathrm{mg}$ twice daily for five days, with a weight-based reduction in the dose in children less than 13 years old) was begun on the day of admission in all patients. The remaining six patients, for whom only specimens obtained at admission and not during treatment were available, presented at similar days of illness (median, six days; range, four to seven) and also began to receive oseltamivir therapy on the day of admission. Five of these patients died within one to eight days after admission (median, three).

Influenza A (H5N1) virus was isolated from throat swabs obtained at admission from six of the seven patients (Patients 2, 3, 5, 6, 7, and 8). Sequence analysis of the neuraminidase genes of these viruses revealed the wild-type $274 \mathrm{H}$ residue alone. Measurements of the viral RNA load in sequential throat specimens showed rapid declines to undetectable levels in four patients who survived, whereas viral RNA was still detectable at the completion of oseltamivir treatment in two patients who died (Patients 3 and 4 in Fig. 3). The remaining patient died during the second day after admission, at which time an increase in the viral RNA load was observed (Patient 2 in Fig. 3). 


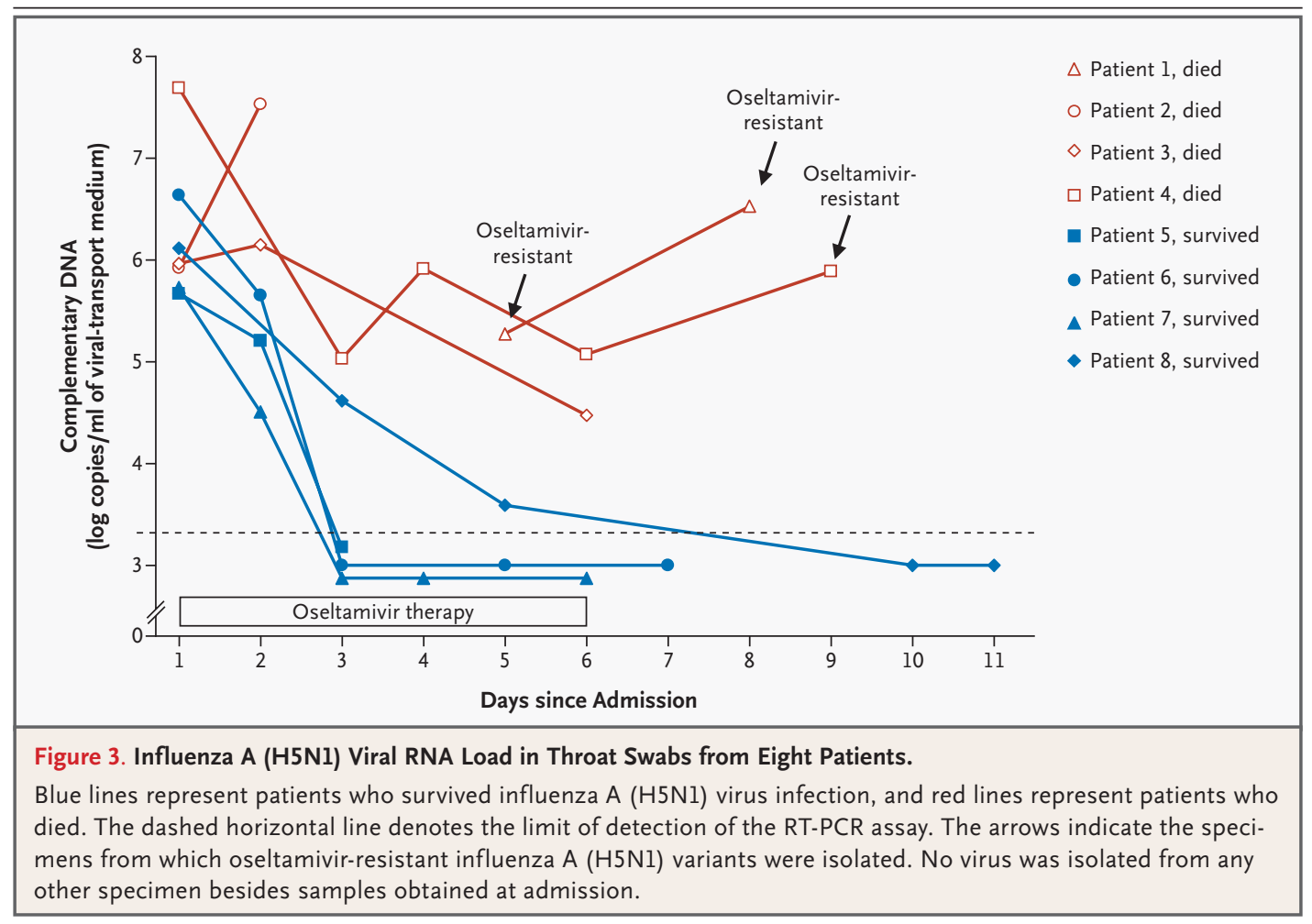

Direct sequencing revealed only wild-type $274 \mathrm{H}$ virus in the second specimen from this patient.

All subsequent throat specimens from Patients 3 through 8 were cultured. Of these specimens, influenza A (H5N1) virus was isolated only from the last specimen from Patient 4, obtained three days after the completion of treatment (Fig. 3). Sequence analysis of this isolate revealed the H274Y substitution in N1. Although sequencing traces of the isolate revealed only mutant $274 \mathrm{Y}$ variants, direct sequencing of viral RNA from the same swab revealed evidence of a minor subpopulation of wild-type $274 \mathrm{H}$ viruses similar to that in specimens from Patient 1 . Patient 4 died of respiratory failure six days after the isolation of resistant virus. Direct sequences of viral RNA from swabs obtained at admission and after two days of treatment showed wild-type $274 \mathrm{H}$ virus alone. The limited sensitivity of the method precluded direct sequencing of further samples from this patient. Likewise, no direct sequences could be obtained from the last specimen obtained from Patient 3.

\section{I S C USS I O N}

We report the isolation from two Vietnamese patients of influenza A (H5N1) viruses with a H274Y substitution in the neuraminidase gene, which confers high-level resistance to oseltamivir. ${ }^{10,11}$ In contrast to the recent report of a partially resistant influenza A (H5N1) virus isolated during once-daily prophylactic treatment with oseltamivir, ${ }^{11}$ the viruses in our patients were isolated during or shortly after a course of oseltamivir at therapeutic twice-daily doses, and mutant $274 \mathrm{Y}$ variants predominated. Furthermore, although the patient with partially resistant virus ultimately received oseltamivir at therapeutic doses and survived, ${ }^{11}$ both of our patients died.

Patient 1 was treated with doses of oseltamivir that were relatively high for her weight, especially during the first day of treatment. Moreover, in this patient, unlike most patients with influenza A (H5N1) virus infection, treatment was started when the greatest clinical benefit could be expected: within 48 hours after the onset of symptoms. Indeed, her clinical condition remained stable during the first three days of treatment without the need for supplemental oxygen. However, on the fourth day of treatment she became progressively dependent on oxygen, her white-cell and platelet counts fell, and there was laboratory evidence of hepatitis. At the time of her death, the viral load in her throat had increased. These observations suggest that the development of drug 
resistance contributed to the failure of therapy and, ultimately, the death of this patient. In the second patient, the viral RNA load declined during treatment, but not to undetectable levels. Whereas only wild-type $274 \mathrm{H}$ virus was detectable after two days of treatment, 274Y mutant virus was isolated shortly after treatment. Although a direct relationship between the emergence of resistance and this patient's death was less clear, the presence of replicating virus after 14 days of illness suggests an effect on the outcome.

The emergence of resistant influenza A (H5N1) variants during oseltamivir treatment should not be surprising. In adults with influenza A (H1N1) or (H3N2) virus infection, the development of resistance to oseltamivir is rare, but resistant viral variants have been detected in up to 18 percent of children who receive oseltamivir. ${ }^{12-14}$ The difference in resistance rates between adults and children may be explained by the occurrence of a primary infection in children associated with higher rates of viral replication owing to a lack of previous immunity. Since humans have no previous immunity to influenza A (H5N1) virus, all human infections with this virus are primary infections. In addition, studies in animals indicate particularly high levels of replication of current influenza A (H5N1) strains. ${ }^{15,16}$

The efficacy of oseltamivir is unlikely to be optimal when treatment is instituted late in the course of illness, as has been the case in most patients with influenza A (H5N1) virus infection reported to date. ${ }^{1,2}$ However, antiviral treatment could still be expected to be beneficial when there is evidence of ongoing viral replication. Such benefit is suggested by the rapid decline in the viral load to undetectable levels in all four survivors in the current series. In contrast, virus was still detectable at the end of treatment in three patients who died of the infection after receiving the full course of treatment, two of whom had oseltamivir-resistant virus isolated from throat specimens. Our observations suggest that at least in some patients with influenza A (H5N1) virus infection, treatment with the recommended dose of oseltamivir incompletely suppresses viral replication. Besides allowing the infection to proceed, such incomplete suppression provides opportunities for drug resistance to develop. Multiple mechanisms may account for inadequate viral suppression by oseltamivir, including overwhelming viral replication, as suggested by the results of studies in mice ${ }^{15}$ and altered pharmacokinetics in severely ill patients, who are apt to have diarrhea. ${ }^{1-3}$ Strategies aimed at improving antiviral efficacy (e.g., the use of higher doses, longer durations of therapy, or combination therapy) may deserve further evaluation. In addition, antiviral agents to which oseltamivir-resistant influenza viruses remain susceptible should be included in treatment arsenals for influenza A (H5N1) virus infections.

Supported by the Wellcome Trust, United Kingdom.

No potential conflict of interest relevant to this article was reported.
REFERENCES

1. The Writing Committee of the World Health Organization (WHO) Consultation on Human Influenza A/H5. Avian influenza A (H5N1) infection in humans. N Eng J Med 2005;353:1374-85.

2. Hien TT, Liem NT, Dung NT, et al. Avian influenza A (H5N1) in 10 patient in Vietnam. N Engl J Med 2004;350:117988.

3. Chotpitayasunondh T, Ungchusak K, Hanshaoworakul W, et al. Human disease from influenza A (H5N1), Thailand, 2004 Emerg Infect Dis 2005;11:201-9.

4. Moscona A. Neuraminidase inhibitors for influenza. N Engl J Med 2005;353:136373.

5. Identification of influenza isolates by hemagglutination inhibition. In: Department of Communicable Disease Surveillance and Response. WHO manual on animal influenza diagnosis and surveillance. Geneva: World Health Organization, 2002: 28-36. (WHO/CDS/CSR/NCS/2002.5.)

6. de Jong MD, Cam BV, Qui PT, et al.

Fatal avian influenza A (H5N1) in a child presenting with diarrhea followed by coma. N Engl J Med 2005;352:686-91.

7. Boom R, Sol C, Beld M, Weel J, Goudsmit J, Wertheim-van Dillen P. Improved silica-guanidiniumthiocyanate DNA isolation procedure based on selective binding of bovine alpha-casein to silica particles. J Clin Microbiol 1999;37:615-9.

8. Boom R, Sol CJ, Salimans MM, Jansen CL, Wertheim-van Dillen PM, van der Noordaa J. Rapid and simple method for purification of nucleic acids. J Clin Microbiol 1990;28:495-503.

9. Chen H, Smith GJ, Zhang SY, et al. Avian flu: H5N1 virus outbreak in migratory waterfowl. Nature 2005;436:191-2.

10. Gubareva LV, Kaiser L, Matrosovich MN, Soo-Hoo Y, Hayden FG. Selection of influenza virus mutants in experimentally infected volunteers treated with oseltamivir. J Infect Dis 2001;183:523-31.

11. Le QM, Kiso M, Someya K, et al. Avian flu: isolation of drug-resistant $\mathrm{H} 5 \mathrm{~N} 1$ virus Nature 2005;437:1108.

12. Whitley RJ, Hayden FG, Reisinger KS, et al. Oral oseltamivir treatment of influenza in children. Pediatr Infect Dis J 2001; 20:127-33. [Erratum, Pediatr Infect Dis J 2001;20:421.]

13. Kiso M, Mitamura K, Sakai-Tagawa Y, et al. Resistant influenza A viruses in children treated with oseltamivir: descriptive study. Lancet 2004;364:759-65.

14. Ward P, Small I, Smith J, Suter P, Dutkowski R. Oseltamivir (Tamiflu) and its potential for use in the event of an influenza pandemic. J Antimicrob Chemother 2005;55:Suppl 1:i5-i21.

15. Yen HL, Monto AS, Webster RG, Govorkova EA. Virulence may determine the necessary duration and dosage of oseltamivir treatment for highly pathogenic A/ Vietnam/1203/04 influenza virus in mice. J Infect Dis 2005;192:665-72.

16. Maines TR, Lu XH, Erb SM, et al. Avian influenza (H5N1) viruses isolated from humans in Asia in 2004 exhibit increased virulence in mammals. J Virol 2005;79: 11788-800.

Copyright (c) 2005 Massachusetts Medical Society. 